\title{
Three-dimensional seismic damage simulation of wooden houses using a rigid body-spring method
}

\author{
H. Kawakami ${ }^{1}$, E. A. Tingatinga ${ }^{2}$ \& H. Y. Chang ${ }^{1}$ \\ ${ }^{1}$ Geoshere Research Institute, Saitama University, Japan \\ ${ }^{2}$ Graduate School of Science and Engineering, Saitama University, Japan
}

\begin{abstract}
In Japan, the majority of modern residential and a fraction of commercial structures are of woodframed construction. The vast number of wooden houses is critical to the infrastructure of the country and their continued good performance is integral to the overall economic welfare. However, the casualties and damage caused by the 1995 Kobe earthquake suggest that these structures are most vulnerable to strong motion earthquakes. Therefore, in order to guarantee the safety of the general public in the event of future earthquakes, it is necessary to study the mechanisms of collapse of these built structures and to provide a way to identify their weak points for the benefit of retrofitting. To address the above issues, a new methodology was developed for the seismic performance assessment of wooden houses. This methodology identifies local failures such as column buckling and connection fracture, which may induce the global system to collapse. In this study, a three-dimensional rigid body-spring method, which can describe the inelastic behaviour of a structure and simulate the progressive collapse process, was employed. The sequence of the analysis and results in the form of computer animations offer a real-time assessment of the structural integrity of buildings during earthquakes.

Keywords: wooden houses, collapse, damage, buildings, rigid body-spring method, simulation.
\end{abstract}

\section{Introduction}

The evolution in computer hardware has had remarkable impact on computing science and engineering design. Desktop and portable computers nowadays are 
operating at tremendous speed, have huge memory resources and multiple processing units, thereby permitting very fast computations never conceived before. Taking advantage of this advancement, several researches have been conducted to simulate various natural phenomena and to analyze engineering problems that were previously infeasible. For the past two decades computer experiments utilizing multimedia and realistic computer animation reinforce, if not replace, traditional expensive experiments.

Recent efforts to simulate earthquake response due to strong motion earthquakes attracted many researchers in the field of architecture, structural and earthquake engineering. One of the most familiar accelerogram used is that of the Kobe earthquake in Japan which occurred in January 17, 1995 and caused enormous damage and destruction of structures and deaths in Kobe and in nearby areas. It claimed more than 6,000 deaths, injured more than 35,000 people and caused about 10 trillion yen worth of damage in Kobe and in nearby areas. The death toll was reported to be mostly caused by the collapse of buildings and breakdown of other civil engineering facilities (Otani [1]). Some were due to landslides, overturning of furniture, fires that broke out resulting from rupturing of gas pipes, etc. However, investigation revealed that most deaths were due to collapse of traditional wooden houses.

In order to guarantee the safety of the general public in the occurrence of future earthquakes, it is necessary to study the mechanisms of collapse of each wooden house and building.

This study presents a three-dimensional simulation of structure collapse during strong motion earthquakes, such as the 1995 Kobe earthquake, using Rigid Body-Spring Method (RBSM). Various modes of collapse of a wooden structure modeled as an assembly of rigid bodies connected by inelastic links at their ends will be presented. In modeling structural components, a link configuration is suggested to take into account structural damping and inelastic behavior.

The main objectives of this study are to simulate the earthquake response of wooden houses, to understand the process in which they collapse, and then to identify the weak point of the structure. Specifically, it aims to

1. simulate collapse mechanisms by modeling structural elements using rigid body-spring method implementing a configuration of inelastic links;

2. investigate how the collapse progresses from the local failures of the framing members.

\section{Nonlinear analysis using assembly of rigid bodies}

Originally proposed by Kawai [2], the basic approach of RBSM is to divide the given structure into appropriate number of rigid elements connected by spring systems. The displacements are completely described by the positions and rotations of the rigid bodies while the deformation energy of the structure is stored in the spring system. 
In this paper, nonlinear analysis of structures will be carried out by introducing nonlinear springs to take into account large displacements and failure of structures during strong motion earthquakes.

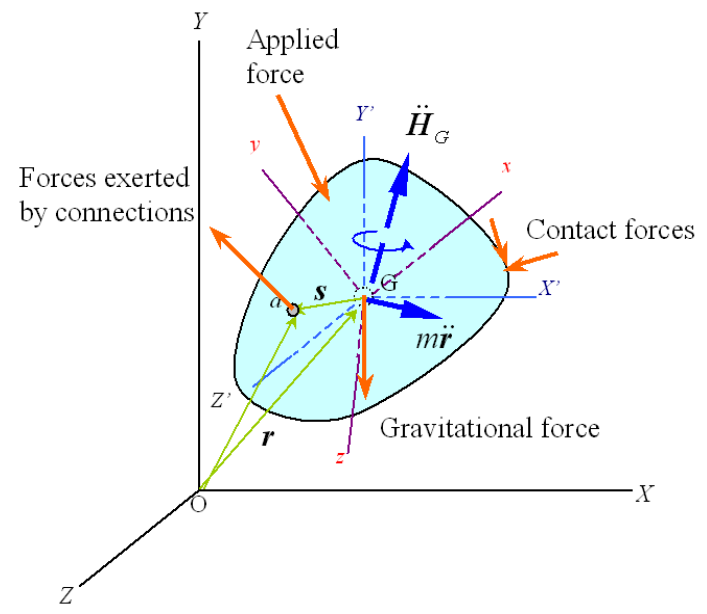

Figure 1: Applied and effective forces on a rigid body and coordinate systems used in computer animation.

\subsection{Position and orientation of bodies [2-4]}

In rigid body assemblies, various coordinates systems must first be well understood (fig.1). A rigid body in space is positioned with respect to the inertial coordinate system OXYZ by a vector $\boldsymbol{r}$ attached to its mass center. Points that define the shape of the body or points where links are attached, say point $\boldsymbol{a}$, are defined by a vector $\boldsymbol{s}$ in terms of local coordinates (with respect to Gxyz). If needed, the global coordinates of this point $S$ are computed as

$$
\boldsymbol{S}=\boldsymbol{R} \boldsymbol{s}+\boldsymbol{r}
$$

where $\boldsymbol{R}$ is a rotation matrix that transforms coordinates from Gxyz frame to OXYZ frame.

Orientation of the body can also be described using Hamilton's quaternion $\boldsymbol{q}$ [4] so that Gxyz is obtained by rotating OXYZ about an axis $\boldsymbol{u}$ by an angle $\gamma$.

$$
\boldsymbol{q}=\left[\cos \frac{\gamma}{2}, \boldsymbol{u} \sin \frac{\gamma}{2}\right]
$$

\subsection{Governing equations [2-4]}

To animate various systems using rigid bodies, appropriate forces must be taken into account. Forces that arise due to relative positioning of objects (e.g., contact, collision), object's velocity, connections (e.g., springs, dampers), and user- 
specified vector fields (e.g., gravity, other external forces) must be exerted on bodies properly. These forces induce linear and angular accelerations depending on the mass and mass distribution of the body, respectively.

The two fundamental equations used to analyze motion of rigid bodies in space are

$$
\begin{aligned}
& \sum \boldsymbol{F}(t)=m \ddot{\boldsymbol{r}}(t) \\
& \sum \boldsymbol{M}_{G}(t)=\dot{\boldsymbol{H}}_{G}(t)
\end{aligned}
$$

where $\ddot{\boldsymbol{r}}(t)$ is the acceleration of the center of mass and $\dot{\boldsymbol{H}}_{G}(t)$ is the rate of change of the angular momentum about the mass center of the rigid body. If $x$, $y$ and $z$ axes coincide with the principal axes of inertia of the rigid body, eqn (4) reduces to the well known Euler's equations of motion and is expressed in terms of the inertia tensor $\sum \boldsymbol{M}_{G}(t)=\boldsymbol{I}(t) \dot{\boldsymbol{\omega}}(t)$.

The above equations can be rewritten by first assuming

$$
\begin{gathered}
\dot{\boldsymbol{r}}(t)=\boldsymbol{v}(t) \\
\dot{\boldsymbol{q}}(t)=\frac{1}{2} \boldsymbol{\omega}(t) * \boldsymbol{q}(t)=\boldsymbol{g}(\boldsymbol{q}(t), \boldsymbol{\omega}(t))
\end{gathered}
$$

where $\boldsymbol{\omega}(t) * \boldsymbol{q}(t)$ denotes a shorthand of the multiplication of two quaternions $[0, \omega(t)]$ and $\boldsymbol{q}(t)$.

At any instant, the state of a rigid body is stored in a vector $\boldsymbol{x}(t)$ consisting of its position, orientation, and its linear and angular velocities (Baraff [4]). Mathematically collected as,

$$
\boldsymbol{x}(t)=\left\{\begin{array}{c}
\boldsymbol{r}(t) \\
\boldsymbol{q}(t) \\
\boldsymbol{v}(t) \\
\boldsymbol{\omega}(t)
\end{array}\right\}_{13 \times 1}
$$

Using eqns (5) through (7), we can rewrite eqns (3) and (4) as the time derivative of the state vector

$$
\dot{\boldsymbol{x}}(t)=\left\{\begin{array}{c}
\boldsymbol{v}(t) \\
\boldsymbol{g}(\boldsymbol{q}(t), \boldsymbol{\omega}(t)) \\
\sum_{-1} \boldsymbol{F}(t) / m \\
\boldsymbol{I}^{-1}(t) \sum \boldsymbol{M}(t)
\end{array}\right\}
$$

This system of first-order differential equations are sufficient to perform physically based animation of rigid bodies. Knowing the current state of the rigid bodies and the derivative information at any time, a differential equation solver can now be used to compute the state vector at a subsequent time. It is important to note that the sum of forces and sum of moments about rigid body's mass center include contact forces and collision forces (or impulses) when it moves relative to another rigid body. This of course entails the use of efficient collision 
detection routines. Since collision detection, response and contact handling are well documented in computer animation and robotics, it will not be discussed in detail in this paper.

\subsection{Earthquake ground motions}

Throughout the scope of this paper, the earthquake accelerogram used was that observed at Kobe Marine Metrological Observatory during of the 1995 Kobe earthquake as shown in fig. 2(a). The maximum accelerations in EW-, NS-, and UD-directions are $6.0 \mathrm{~m} / \mathrm{s}^{2}$ at $5.5 \mathrm{~s}, 8.2 \mathrm{~m} / \mathrm{s}^{2}$ at $5.5 \mathrm{~s}$, and $3.3 \mathrm{~m} / \mathrm{s}^{2}$ at $4.7 \mathrm{~s}$, respectively. Figure 2(b) shows the corresponding displacement-time histories.

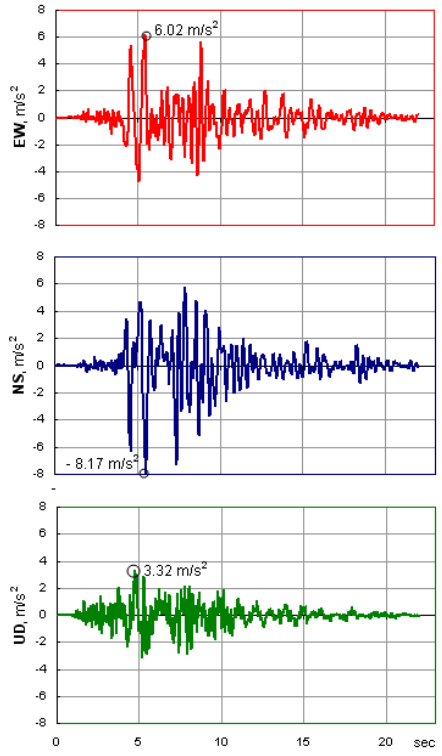

(a)
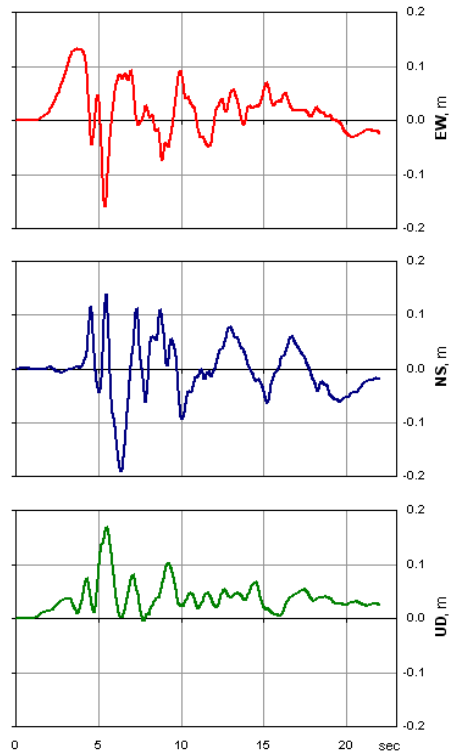

(b)

Figure 2: Time history of (a) ground acceleration and (b) ground displacement of the 1995 Kobe earthquake used in analyses.

\subsection{System of non-linear springs and dashpots}

A new link consisting of a spring and a damper, parallel to one another is introduced. Forces exerted to points on connected bodies is the vector sum of spring and damper components. The stress-strain behavior of materials, as modeled by springs, is idealized by the straight lines in fig. 3 . In this model the restoring force in tension is proportional to the strain up to $\varepsilon_{Y T}$ with maximum yield restoring force $F_{Y T}$. The second straight line represents the strainhardening characteristic until point $\mathrm{C}$, when the restoring force reaches its 
ultimate value $F_{U T}$. For different set of parameters $\varepsilon_{Y C}, F_{Y C}, \varepsilon_{U C}$, and $F_{U C}$, the behavior in compression can be modeled. If the strain in the link exceeds its maximum value $\varepsilon_{U T}$ or $\varepsilon_{U C}$, no subsequent forces are exerted to the connected bodies. The link is marked DELETED to aid analysis of link failure. A set of links are positioned to model axial, shear and bending deformations.

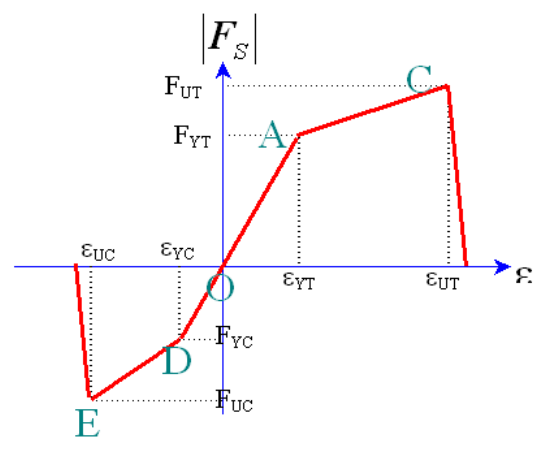

Figure 3: $\quad$ Force exerted by link on rigid bodies.

\section{Application to wooden houses [6]}

\subsection{Dynamic collapse of conventional wooden houses}

Using the numerical method presented above, earthquake responses of typical wooden houses in Japan, as shown in fig. 4, were computed. In modeling, the dead load of the floor slab, beams, columns, walls and roofs, and the live loads were estimated. The stress-strain curves for the material of the structural members were modeled. The model structures were then subjected to doublyamplified waves of the 1995 Kobe earthquake in fig. 2.

Figures 4(a)-(d) show various collapse mechanisms of wooden houses including (a) collapse due to the soft first story, (b) tumbling type collapse, (c) failure at the second floor due to the amplification of the vibration at the upper floor, and (d) collapse of intermediate floor.

Even though the soft first story type of collapse is often stressed, the failure mechanism depends on the design and physical layout of the structure, i.e., strength and distributions of columns, beams and walls. For example, fig. 5 shows the response sequence of the three-storey wooden house in fig. 4(d). During the strong motion earthquake, the house deformed beyond the limit of linearly elastic behavior, and the second floor began to twist at around $4 \mathrm{~s}$, and collapsed at about $6 \mathrm{~s}$.

\subsection{Reliability of simulated response}

Most wooden houses in Japan are generally composed of frame units made of columns and beams, and walls. Therefore, in order to establish the reliability and correctness of collapse simulation of an entire house, it is obligatory to show the 
agreement of simulated and experimental results for such structural units. Figure 6 shows frames with different types of reinforcements. A monotonically increasing force was then applied horizontally to the upper beam of each frame, and the response was computed using the same program that was used in figs. 4 and 5 .

(a)

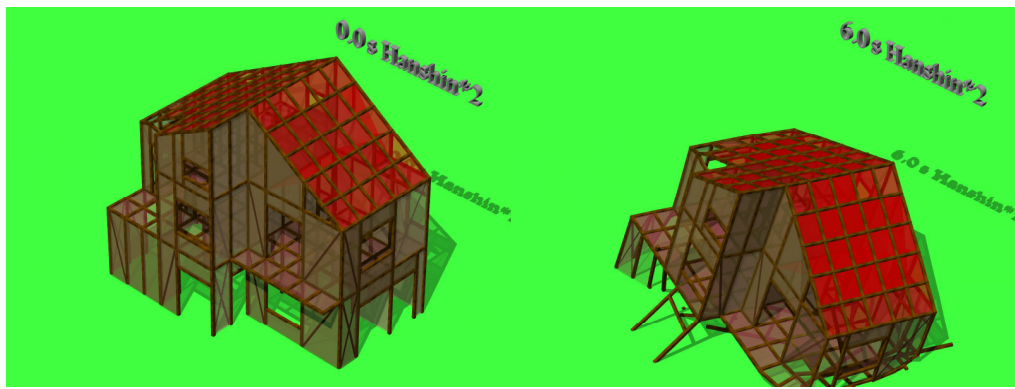

(b)

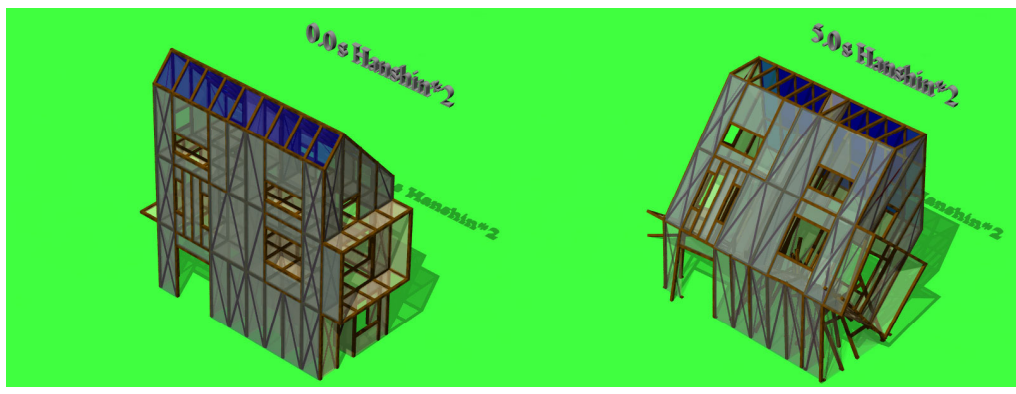

(c)

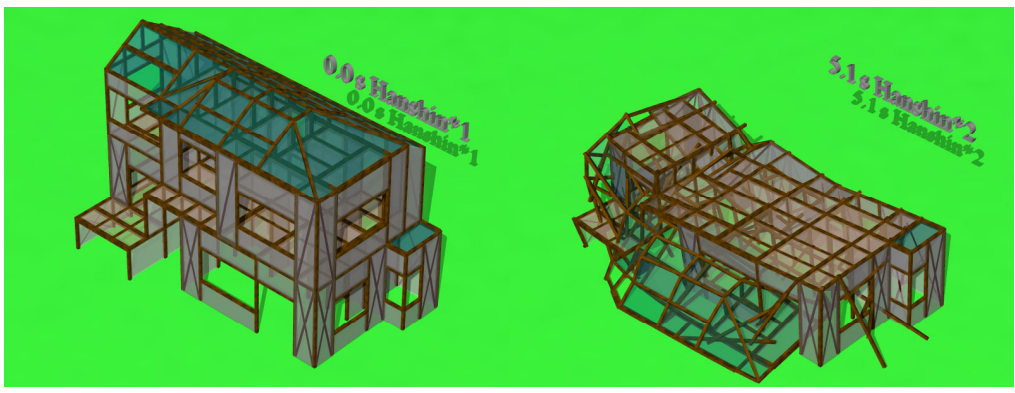

(d)

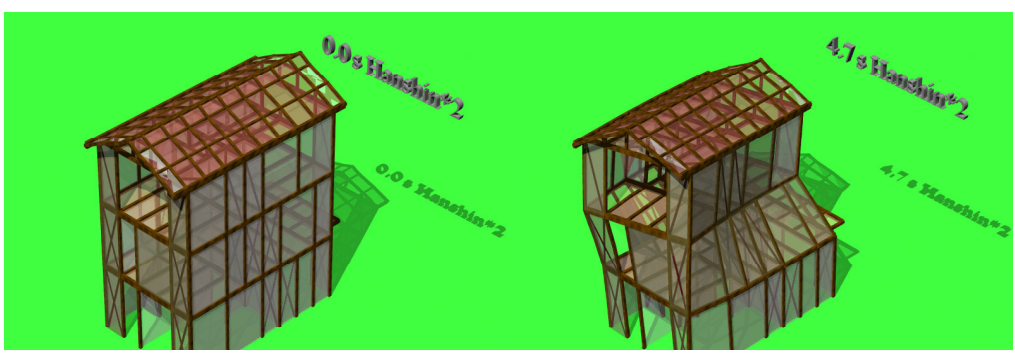

Figure 4: Collapse mechanisms of typical wooden houses. 


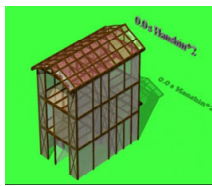

0.0

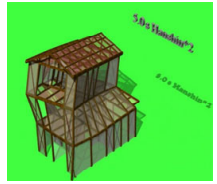

5.0

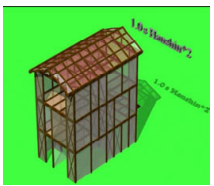

1.0

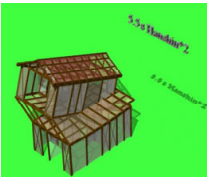

5.5

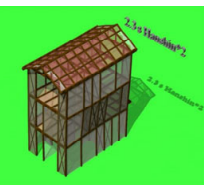

2.3

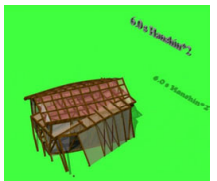

6.0

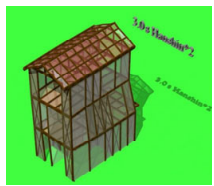

3.0

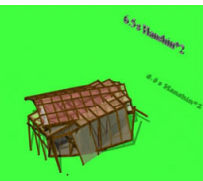

6.5

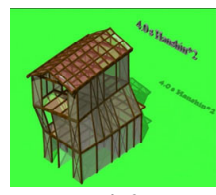

$4.0 \mathrm{~s}$

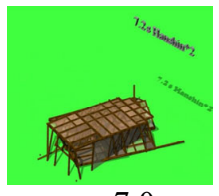

$7.0 \mathrm{~s}$

Figure 5: $\quad$ Progressive collapse sequence of a three-storey wooden house.

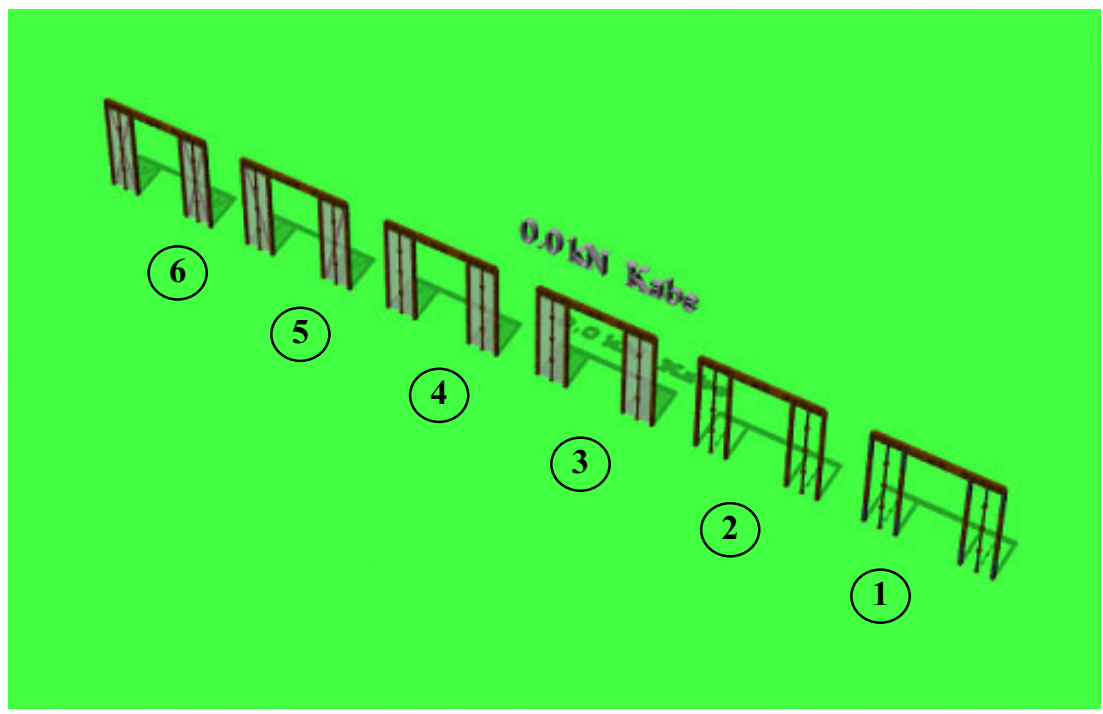

Figure 6: Samples of wooden frame units. From right to left: wooden frames with (1) no end connection plate; (2) end connection plates; (3) plaster board; (4) siding board; (5) two single braces, plaster board and siding board; and (6) two double braces, plaster board and siding board.

Based on the obtained response of the upper beam, the relationship between the displacement and force was plotted by solid lines in fig. 7 for each frame unit (1) through (6). The experimental relationships obtained by Miyoshi et al. [5] were also plotted by dashed lines in fig. 7. The numerical and the experimental curves agree well including the rigidity in small strain, the maximum strength, and the weakening process, and this shows the reliability of the simulation in this paper. 


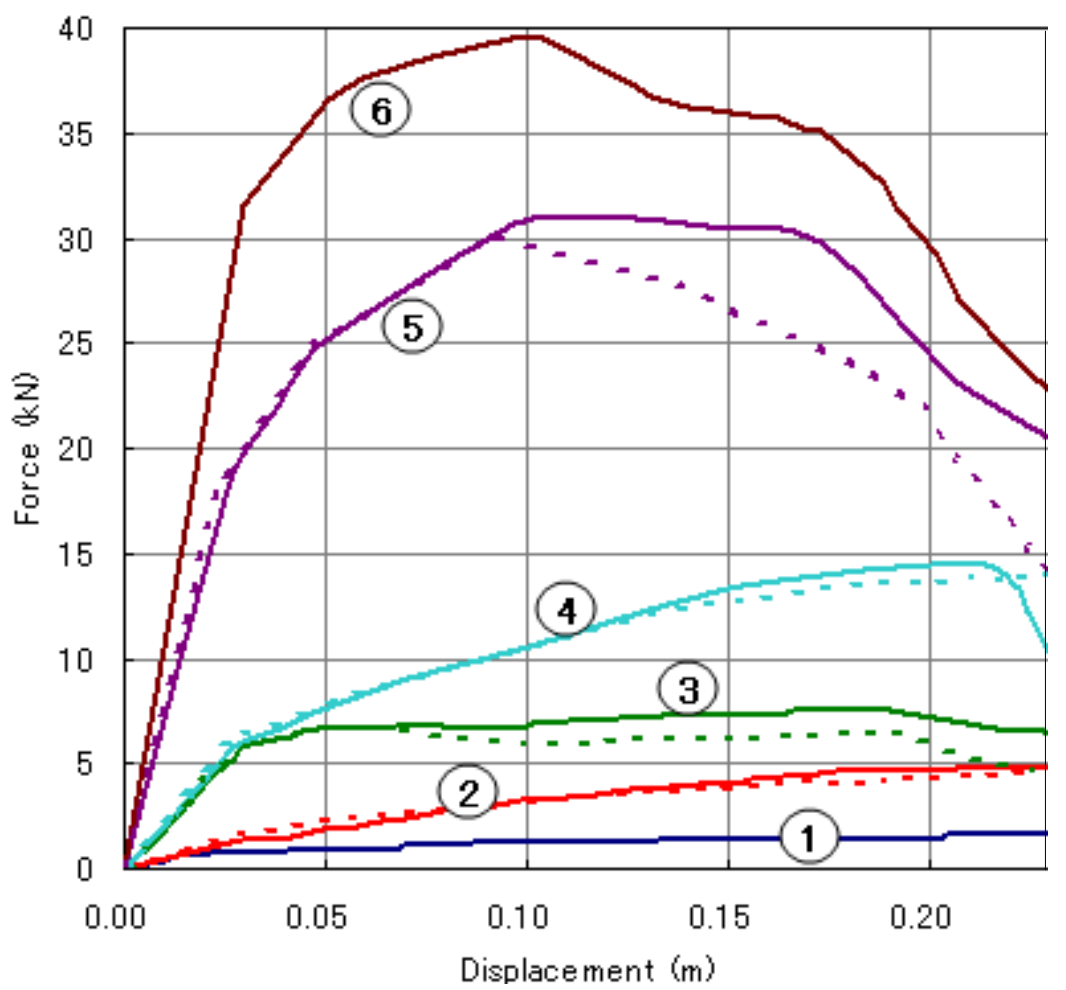

Figure 7: $\quad$ Force-displacement relationships of wooden frames.

\section{Conclusions}

This research attempted to simulate seismic collapse of wooden houses subjected to the 1995 Kobe earthquake using the Rigid Body-Spring Method (RBSM), and the following conclusions were drawn:

1. The simplified model of wooden houses is capable of demonstrating to some extent various collapse behavior during strong motion earthquakes. More accurately, the link system used to characterize plastic hinges can simulate local failure that causes the entire house to collapse during strong motion earthquakes.

2. The method provides a way to identify the weak point of a structure thus allowing engineers to perform retrofitting analysis easily so as to suggest ways to improve the seismic performance of built wooden houses.

\section{References}

[1] Otani, S., Disaster mitigation engineering -the Kobe earthquake disaster-, JSPS Seminar on Engineering in Japan, Royal Society, London, 1999. 
[2] Kawai, T., New element models in discrete structural analysis, Japan Society of Naval Architects, Japan, 141, pp. 174-180, 1977.

[3] Hamilton, W. R., On quaternions, Proceedings of the Royal Irish Academy, 3, pp. 1-16, 1847.

[4] Baraff, D., Dynamic Simulation of Non-Penetrating Rigid Bodies, Ph.D. Thesis, Cornell University, 1992.

[5] Miyoshi, K., Ohashi, Y., Takahashi, K., Watahiki, M. \& Nakano, I., Static loading test and shaking table test of walls for wooden houses, Part 1: static loading test, Summaries of Technical Papers of Annual Meeting, Architectural Institute of Japan, pp. 199-200, 2001 (in Japanese).

[6] Kawakami, H., www.saitama-u.ac.jp/kawakami/ (in Japanese). 